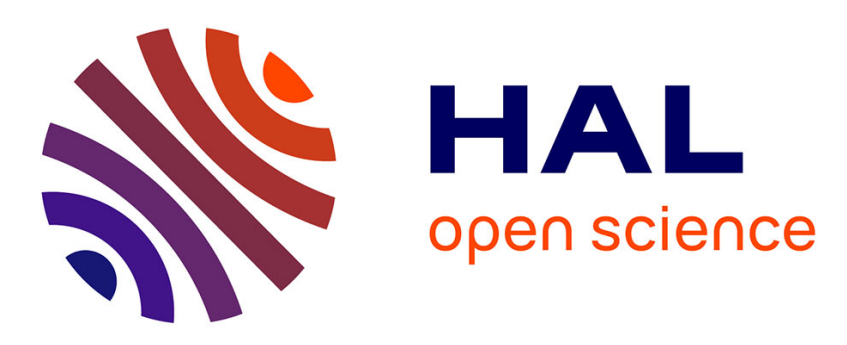

\title{
Leader-follower consensus formation control of differential-drive nonholonomic vehicles with time-varying delays
}

\author{
Antonio Loria, Emmanuel Nuño, Elena Panteley
}

\section{- To cite this version:}

Antonio Loria, Emmanuel Nuño, Elena Panteley. Leader-follower consensus formation control of differential-drive nonholonomic vehicles with time-varying delays. 2021 29th Mediterranean Conference on Control and Automation (MED), Jun 2021, PUGLIA, Italy. pp.1240-1245, 10.1109/MED51440.2021.9480222 . hal-03442742

HAL Id: hal-03442742

https://hal.science/hal-03442742

Submitted on 23 Nov 2021

HAL is a multi-disciplinary open access archive for the deposit and dissemination of scientific research documents, whether they are published or not. The documents may come from teaching and research institutions in France or abroad, or from public or private research centers.
L'archive ouverte pluridisciplinaire HAL, est destinée au dépôt et à la diffusion de documents scientifiques de niveau recherche, publiés ou non, émanant des établissements d'enseignement et de recherche français ou étrangers, des laboratoires publics ou privés. 


\title{
Leader-follower consensus formation control of differential-drive nonholonomic vehicles with time-varying delays
}

\author{
Antonio Loría Emmanuel Nuño Elena Panteley
}

\begin{abstract}
In this paper is addressed the problem of formation set-point control of mobile robots, exchanging information over a network affected by communication delays. It is assumed, moreover, that the latter may be time-varying which is commonly the case of wireless networks. On the other hand, the autonomous robots are modelled as second-order mechanical systems with nonholonomic constraints; in particular, as differential-drive vehicles. The control objective is to ensure that all the robots coordinate their motion and acquire a formation pattern located at a desired position. Furthermore, they are all required to synchronize their orientations to the leader's. The control approach exploits the structural properties of the system. Being second-order mechanical systems, we design decentralized controllers of the proportional-derivative (PD) type, endowed with a time-varying vanishing term that excites all the system's modes to render the whole space reachable in spite of the nonholonomic constraints (nonholonomic systems are not stabilizable to a point via smooth time-invariant feedback). We provide theoretical justification of our main result and illustrate our findings via numerical simulations.
\end{abstract}

\section{INTRODUCTION}

The control objective in the consensus of multiple dynamical agents is to ensure that the states of all agents agree on a common value by sharing their state with their corresponding neighbors [1]. There are mainly two different consensus problems in dynamical systems: the leaderfollower, in which the agreement value is given as a desired value to a set of (follower) agents in the network; and the leaderless, in which such common value is completely determined by the initial conditions of all agents in the network [2], [3], [4]. For autonomous vehicles, which can obviously not occupy the same physical space, the formation consensus problem consists in making the positions of all robots converge to a common value, modulo an offset, i.e., a vector originating at the formation's center which determines a predefined position of the robot with respect to that center. This is the problem addressed in this paper. Other formation control problems consist in making the robots follow a

Emmanuel Nuño is with the Department of Computer Science at the University of Guadalajara. Guadalajara, Mexico (email: emmanuel.nuno@cucei.udg.mx). Antonio Loría and Elena Panteley are with the CNRS, at Laboratoire des Signaux et Systèmes, L2S-CentraleSupélec, Gif-sur-Yvette, France; (e-mail: \{antonio.loria\}\{elena.panteley\}@centralesupelec.fr).

This work has been partially supported by the Mexican CONACyT Basic Scientific Research grant CB-282807, by Government of Russian Federation (grant 074-U01), by the French National Research Agency (ANR) via the project "HANDY" — contract number: ANR-18-CE40-0010, and by CEFIPRA under the grant number 6001-A moving target, these are referred to as formation-tracking and are fundamentally different in that the reference trajectory for the swarm is time-varying. Indeed, for nonholonomic systems, set-point stabilization is not a particular case of tracking control [5]. For instance, in [6] the problem of formation-tracking control is addressed in a way that all robots have access to the virtual reference robot, but consensus per se is not investigated. Also, only the linear-motion dynamics is controlled - orientations are neglected. Indeed, in certain scenarii, as the one addressed here, it may be required that all robots acquire a common orientation or each a different reference angle. For other classifications of formation control problems of autonomous vehicles see [7].

Consensus of nonholonomic mobile robots has been studied, for instance, in [8] where a decentralized feedback control that drives a system of multiple nonholonomic unicycles to a rendezvous point in terms of both position and orientation is proposed, the control law is discontinuous and time-invariant. In [9] necessary and sufficient conditions for the feasibility of a class of position formations are laid. In [10] the position/orientation formation control problem for multiple nonholonomic agents using a timevarying controller that leads the agents to a given formation using only their orientation is proposed. In [11] a distributed consensus control law for a network of nonholonomic agents in the presence of bounded disturbances with unknown dynamics in all inputs channels is presented. In [12] a smooth distributed formation control law using a consensusbased approach to drive a group of agents to a desired geometric pattern is proposed. The latter result is extended in [13] by introducing a proportional derivative controller for the velocity dynamics. In the previously mentioned references, however, it is assumed that the communications are completely reliable and do not exhibit time-delays. In addition, a simplified kinematics model of the nonholonomic mobile robots is used. In [14] and in [15] a more realistic torque-controlled second-order dynamics model is used.

In this article we address the leader-follower consensus problem, with constant desired position and orientation, for nonholonomic mobile robots under the realistic scenario that the network is affected by time-varying delays. Although this problem has been studied, for instance in [16], it is assumed therein that the delays are constant. The proposed controller is smooth and decentralized. It consists in two simple-to-implement proportional-derivative controllers, for the linear and angular motion dynamics respectively, and an additional nonlinear time-varying function is added to cope 
with the motion restrictions imposed by the nonholonomic constraints.

The rest of the paper is organized as follows. In the next section we present the dynamic model and the problem formulation. Our main result is provided in Section III and illustrative numerical simulations are provided in Section IV. The paper is wrapped up with some concluding remarks.

\section{Problem Setting And Dynamical Model}

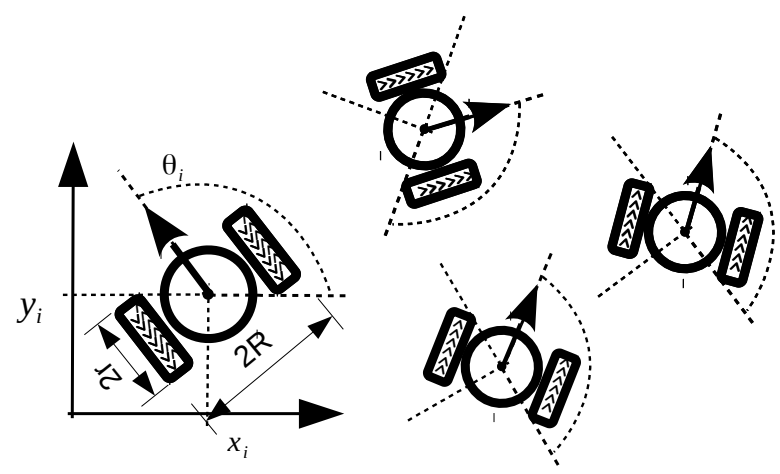

Fig. 1. Schematical representation of a group of differential-drive robots

We consider a swarm of $N$ nonholonomic vehicles modeled as differential drive robots that move in the Cartesian $x y$-plane with three degrees of freedom, two translations and one rotation. Let us define $\mathbf{z}_{i}:=\left[\begin{array}{ll}x_{i} & y_{i}\end{array}\right]^{\top} \in \mathbb{R}^{2}$ and $\theta_{i} \in \mathbb{R}$ as the translation and rotation coordinates, respectively, of the $i$ th-robot and $i \in \bar{N}:=\{1, \ldots, N\}$. Let us also define $\boldsymbol{\delta}_{i} \in \mathbb{R}^{2}$ as the relative (constant) desired translation of the $i$ th-robot with regards to the barycenter of a given formation pattern. Thus, the corresponding position of the $i$ th-robot translated to the barycenter of the desired formation is $\overline{\mathbf{z}}_{i}:=\mathbf{z}_{i}-\boldsymbol{\delta}_{i}$. In the rest of the paper we consider that $i \in \bar{N}$.

Remark 1 A desired orientation of the formation pattern may also be also imposed by setting $\overline{\mathbf{z}}_{i}:=\mathbf{z}_{i}-\boldsymbol{\delta}_{i}^{*}$, where $\boldsymbol{\delta}_{i}^{*}$ is given by

$$
\boldsymbol{\delta}_{i}^{*}=\left[\begin{array}{cc}
\cos \left(\phi_{d}\right) & -\sin \left(\phi_{d}\right) \\
\sin \left(\phi_{d}\right) & \cos \left(\phi_{d}\right)
\end{array}\right] \boldsymbol{\delta}_{i}
$$

where $\phi_{d} \in \mathbb{R}$ is the (constant) desired formation pattern orientation.

\section{A. Interconnection Topology}

It is assumed that each robot communicates bilaterally with a set of neighbours $\mathcal{N}_{i}$ over a wireless network. Their interconnections are modelled using the Laplacian matrix $\mathbf{L}:=\left[\ell_{i j}\right] \in \mathbb{R}^{N \times N}$, whose elements are defined as

$$
\ell_{i j}=\left\{\begin{array}{cc}
\sum_{k \in \mathcal{N}_{i}} a_{i k} & i=k \\
-a_{i k} & i \neq k
\end{array}\right.
$$

where $a_{i k}>0$ if $k \in \mathcal{N}_{i}$ and $a_{i k}=0$ otherwise. It is assumed that
(A1) the interconnection graph is undirected, static and connected.

Therefore, by construction, $\mathbf{L}$ has a zero row sum, i.e., $\mathbf{L} \mathbf{1}_{N}=\mathbf{0}_{N}$. Moreover, Assumption A1, ensures that $\mathbf{L}$ is symmetric, has a single zero-eigenvalue and the rest of the spectrum of $\mathbf{L}$ is strictly positive. Thus, $\operatorname{rank}(\mathbf{L})=N-1$. Therefore, the vectors belonging to the kernel of $\mathbf{L}$ belong to $\operatorname{span}\left\{\mathbf{1}_{N}\right\}$.

Furthermore we assume that

(A2) there is a non-empty set of robots that has direct access to the desired position of the barycenter of the formation $\mathbf{z}_{\ell} \in \mathbb{R}^{2}$ and the desired orientation, $\theta_{\ell}$.

Assumption $\mathbf{A 2}$ ensures that there exists at least $i \in \bar{N}$ such that $b_{i}>0$. Then, let us define a diagonal matrix $\mathbf{B} \in \mathbb{R}^{N \times N}$ to model the leader-follower interconnections. The following lemma, which is a special case of Lemma 3 in [17] and Lemma 1.6 of [2], provides a fundamental property of the composed Laplacian matrix $\mathbf{L}_{\ell}:=\mathbf{L}+\mathbf{B}$.

Lemma 1 Consider a non-negative diagonal matrix $\mathbf{B}:=$ $\operatorname{diag}\left\{b_{i}\right\} \in \mathbb{R}^{N \times N}$ and suppose that at least one $b_{i}$ is strictly positive. Assume that $\mathbf{A} \mathbf{1}$ holds, then $\mathbf{L}_{\ell}:=\mathbf{L}+\mathbf{B}$ is symmetric, positive definite and of full rank.

It is further assumed that the information exchange between the vehicles is subjected to time-delays that satisfy the following assumption.

(A3) The communication from the $j$ th to the $i$ th robot is subject to a variable time-delay denoted $T_{i j}(t)$ which is bounded by a known upper-bound $T_{i j}^{*} \geq 0$. Further, $T_{i j}(t)$ has bounded time-derivatives.

\section{B. Control Objective}

The control objective is to design a decentralized controller for a swarm of $N$ nonholonomic vehicles modeled as differential drive robots, for which Assumptions A1-A3 hold, to solve the following problem:

Leader-Follower Formation Problem. Given a desired formation pattern, i.e., given $\boldsymbol{\delta}_{i} \in \mathbb{R}^{2}$, a (constant) desired barycenter formation position $\mathbf{z}_{\ell} \in \mathbb{R}^{2}$, and a (constant) leader orientation $\theta_{\ell} \in \mathbb{R}$ which is accessible only to some, but not necessarily to all the robots, design a decentralized controller that ensures that all the relative Cartesian positions and orientations of the robots (globally and asymptotically) converge to each other and to the desired values, i.e., for all $\mathbf{z}_{i} \in \mathbb{R}^{2}$ and $\theta_{i} \in \mathbb{R}$

$$
\lim _{t \rightarrow \infty}\left[\begin{array}{c}
\overline{\mathbf{z}}_{i}(t) \\
\theta_{i}(t)
\end{array}\right]=\left[\begin{array}{c}
\mathbf{z}_{\ell} \\
\theta_{\ell}
\end{array}\right],
$$

where $\overline{\mathbf{z}}_{i}:=\mathbf{z}_{i}-\delta_{i}$.

\section{Dynamic Model}

We assume that for each robot, the geometrical center and the center of mass are located at the same point $\mathbf{z}_{i}:=$ 
$\left[\begin{array}{ll}x_{i} & y_{i}\end{array}\right]^{\top}$. Then, the corresponding kinematics of the $i$ th-robot is given by

$$
\begin{aligned}
\dot{\overline{\mathbf{z}}}_{i} & =\boldsymbol{\varphi}_{i} v_{i}, \\
\dot{\theta}_{i} & =\omega_{i}
\end{aligned}
$$

and its dynamics is given by

$$
\left[\begin{array}{c}
\dot{v}_{i} \\
\dot{\omega}_{i}
\end{array}\right]=\left[\begin{array}{cc}
\frac{1}{m_{i}} & 0 \\
0 & \frac{1}{I_{i}}
\end{array}\right] \mathbf{M}_{i} \boldsymbol{\tau}_{i}
$$

where $\varphi_{i}=\left[\begin{array}{ll}\cos \left(\theta_{i}\right) & \sin \left(\theta_{i}\right)\end{array}\right]^{\top} ; v_{i}$ and $\omega_{i}$ are the linear and the angular velocities of the center of mass, respectively; $m_{i}$ is the mass; $I_{i}$ is the moment of inertia; $r_{i}$ the radius of the wheels; and $R_{i}$ is the distance between point $\mathbf{z}_{i}$ and the wheels. $\boldsymbol{\tau}_{i}$ is the control input torque of the left and right wheels, i.e., $\boldsymbol{\tau}_{i}=\left[\tau_{i l}, \tau_{i r}\right]^{\top}$, and $\mathbf{M}_{i}=\frac{1}{r_{i}}\left[\begin{array}{cc}1 & 1 \\ R_{i} & -R_{i}\end{array}\right]$ -see Fig. 1.

A first step in the controller design is to propose the following inner control-loop

$$
\boldsymbol{\tau}_{i}=\mathbf{M}_{i}^{-1} \mathbf{u}_{i}=\frac{r_{i}}{2}\left[\begin{array}{cc}
1 & \frac{1}{R_{i}} \\
1 & -\frac{1}{R_{i}}
\end{array}\right]\left[\begin{array}{l}
u_{v i} \\
u_{\omega i}
\end{array}\right]
$$

where the extra input term $\mathbf{u}_{i} \in \mathbb{R}^{2}$ will be defined in the next section. Then, the closed-loop (3) and (4) has the structure of two second-order mechanical systems, one that corresponds to the linear-motion dynamics and another to the angular-motion. That is,

$$
\begin{aligned}
& \text { linear motion : }\left\{\begin{aligned}
\dot{\overline{\mathbf{z}}}_{i} & =\boldsymbol{\varphi}_{i}\left(\theta_{i}\right) v_{i} \\
\dot{v}_{i} & =-\frac{1}{m_{i}} u_{v i}
\end{aligned}\right. \\
& \text { angular motion : }\left\{\begin{aligned}
\dot{\theta}_{i} & =\omega_{i} \\
\dot{\omega}_{i} & =-\frac{1}{I_{i}} u_{\omega i}
\end{aligned}\right.
\end{aligned}
$$

Then, we design control laws for these equations.

\section{MAIN RESULT}

We solve the previously formulated problem using a smooth controller that is composed of two parts, one for the linear motion and another for the angular motion. The linearmotion control input is designed as a simple proportionalderivative (PD) controller, given by

$$
u_{v i}=-p_{v i} b_{i} \boldsymbol{\varphi}_{i}^{\top}\left(\overline{\mathbf{z}}_{i}-\overline{\mathbf{z}}_{\ell}\right)-p_{v i} \boldsymbol{\varphi}_{i}^{\top} \mathbf{e}_{z i}-d_{v i} v_{i},
$$

where $p_{v i}$ and $d_{v i}>0$ are the proportional and the damping gains respectively while the gain $b_{i}$ is positive if the leader (desired) barycenter of the formation, $\overline{\mathbf{z}}_{\ell}$, is available to the $i$ th-robot and $b_{i}=0$ otherwise. The variable $\mathbf{e}_{z i}$ denotes the $i$ th Cartesian position error, which is defined as

$$
\mathbf{e}_{z i}:=\sum_{j \in \mathcal{N}_{i}} a_{i j}\left(\overline{\mathbf{z}}_{i}-\overline{\mathbf{z}}_{j}\left(t-T_{i j}(t)\right)\right)
$$

It is to be noted that the proportional terms are also premultiplied by the vector $\varphi_{i}\left(\theta_{i}\right)$ to account for the system's kinematics.
The angular-motion control input is also of the PD type, but an extra time-varying input is added to cope with the nonholonomic constraints ${ }^{1}$. More precisely, we pose

$$
u_{\omega i}=-p_{\omega i} b_{i}\left(\theta_{i}-\theta_{\ell}\right)-p_{\omega i} e_{\theta i}-d_{\omega i} \omega_{i}+\gamma_{i}\left(t, \theta_{i}, \mathbf{e}_{z i}\right)
$$

in which the orientation consensus error $e_{\theta i}$ is given by

$$
e_{\theta i}:=\sum_{j \in \mathcal{N}_{i}} a_{i j}\left(\theta_{i}-\theta_{j}\left(t-T_{i j}(t)\right)\right)
$$

and $\gamma_{i}$, which is given by

$$
\gamma_{i}\left(t, \theta_{i}, \mathbf{e}_{z i}\right):=k_{\gamma i} f_{i}(t) \varphi_{i}^{\perp \top}\left[b_{i}\left(\overline{\mathbf{z}}_{i}-\overline{\mathbf{z}}_{\ell}\right)+\mathbf{e}_{z i}\right],
$$

is added to introduce persistency of excitation to the controller as long as the consensus errors are different from zero - $f$. [18]. To that end, $f_{i}(t)$ is defined as any sufficiently smooth function such that $f_{i}, \dot{f}_{i}, \ddot{f}_{i} \in \mathcal{L}_{\infty}, \lim _{t \rightarrow \infty} f_{i}(t) \neq 0$ and $\lim _{t \rightarrow \infty} \dot{f}_{i}(t) \neq 0$. Furthermore, the function $\varphi_{i}^{\perp}$ is the annihilator of $\boldsymbol{\varphi}_{i}$, i.e., $\boldsymbol{\varphi}_{i}^{\top} \boldsymbol{\varphi}_{i}^{\perp}=\boldsymbol{\varphi}_{i}^{\perp \top} \boldsymbol{\varphi}_{i}=0$, and it is given by $\varphi_{i}^{\perp}=\left[\begin{array}{ll}-\sin \left(\theta_{i}\right) & \cos \left(\theta_{i}\right)\end{array}\right]^{\top}$. The control gain $k_{\gamma i}>0$ and

Our main statement, presented next, is that the LeaderFollower Formation Problem is solved via the controller introduced above.

Proposition 1 Suppose that Assumptions A1, A2, and A3 hold for the systems (2)-(4). Then, the controller defined by (7)-(11) solves the (LF) problem, provided that the damping gains $d_{v i}$ and $d_{\omega i}$ satisfy

$$
\begin{aligned}
d_{v i} & >\frac{1}{2} p_{v i} \sum_{j \in \mathcal{N}_{i}} a_{i j}\left(\beta_{i}+\frac{T_{i j}^{* 2}}{\beta_{j}}\right) \\
d_{\omega i} & >\frac{1}{2} p_{\omega i} \sum_{j \in \mathcal{N}_{i}} a_{i j}\left(\alpha_{i}+\frac{T_{i j}^{* 2}}{\delta_{j}}\right)
\end{aligned}
$$

for some $\alpha_{i}, \beta_{i}>0$ for all $i \in \bar{N}$.

Proof of Proposition 1. The linear-motion closed-loop dynamics resulting from using (7) in (5a) and recalling (2a), yields

$$
\Sigma_{v}\left\{\begin{aligned}
\dot{\mathbf{z}}_{i} & =\boldsymbol{\varphi}_{i} v_{i} \\
\dot{v}_{i} & =-\frac{p_{v i}}{m_{i}}\left[b_{i} \boldsymbol{\varphi}_{i}^{\top}\left(\overline{\mathbf{z}}_{i}-\overline{\mathbf{z}}_{\ell}\right)+\boldsymbol{\varphi}_{i}^{\top} \mathbf{e}_{z i}+\frac{d_{v i}}{p_{v i}} v_{i}\right] .
\end{aligned}\right.
$$

while the closed-loop system corresponding to the angular motion, results from replacing (9) in (6b) and recalling (2b). That is

$$
\Sigma_{\omega}\left\{\begin{array}{l}
\dot{\theta}_{i}=\omega_{i} \\
\dot{\omega}_{i}=-\frac{p_{\omega i}}{I_{i}}\left[b_{i}\left(\theta_{i}-\theta_{\ell}\right)+e_{\theta i}+\frac{d_{\omega i}}{p_{\omega i}} \omega_{i}-\frac{1}{p_{\omega i}} \gamma_{i}\right]
\end{array}\right.
$$

${ }^{1}$ The problem under study here is inherently of set-point stabilization, but nonholonomic systems are not stabilizable to a point via smooth timeinvariant feedback. 
The analysis of these sets of equations is divided in two stages. First, we establish boundedness of all trajectories and then we show that all error trajectories converge to zero.

Stage 1a) Boundedness of $\overline{\mathbf{z}}_{i}(t)-\overline{\mathbf{z}}_{j}(t)$ and $v_{i}(t)$ : Consider the Lyapunov-Krasovskii functional

$$
\begin{aligned}
\mathcal{V}:=\frac{1}{2} \sum_{i \in \bar{N}} b_{i}\left|\overline{\mathbf{z}}_{i}-\overline{\mathbf{z}}_{\ell}\right|^{2}+ & \\
\sum_{i \in \bar{N}} & {\left[\frac{m_{i}}{2 p_{v i}} v_{i}^{2}+\frac{1}{4} \sum_{j \in \mathcal{N}_{i}} a_{i j}\left|\overline{\mathbf{z}}_{i}-\overline{\mathbf{z}}_{j}\right|^{2}\right.} \\
& \left.+\frac{1}{2 \beta_{i}} \sum_{j \in \mathcal{N}_{i}} a_{i j} T_{i j}^{*} \int_{-T_{i j}^{*}}^{0} \int_{t+\sigma}^{t} v_{j}^{2}(\eta) d \eta d \sigma\right] .
\end{aligned}
$$

Then, evaluating the total derivative of $\mathcal{V}$ along the trajectories of $\Sigma_{v}$ in (14) yields

$$
\begin{aligned}
& \dot{\mathcal{V}}=-\sum_{i \in \bar{N}}\left[\frac{d_{v i}}{p_{v i}} v_{i}^{2}+\dot{\overline{\mathbf{z}}}_{i}^{\top} \sum_{j \in \mathcal{N}_{i}} a_{i j}\left(\overline{\mathbf{z}}_{i}-\overline{\mathbf{z}}_{j}\left(t-T_{i j}(t)\right)\right)\right. \\
& -\frac{1}{2} \sum_{j \in \mathcal{N}_{i}} a_{i j}\left(\dot{\overline{\mathbf{z}}}_{i}-\dot{\overline{\mathbf{z}}}_{j}\right)^{\top}\left(\overline{\mathbf{z}}_{i}-\overline{\mathbf{z}}_{j}\right) \\
& \left.-\frac{1}{2 \beta_{i}} \sum_{j \in \mathcal{N}_{i}} a_{i j}\left(T_{i j}^{* 2} v_{j}^{2}-T_{i j}^{*} \int_{t-T_{i j}^{*}}^{t} v_{j}^{2}(\sigma) d \sigma\right)\right] . \\
& =-\sum_{i \in \bar{N}}\left[\frac{d_{v i}}{p_{v i}} v_{i}^{2}+\sum_{j \in \mathcal{N}_{i}} a_{i j} \dot{\overline{\mathbf{z}}}_{i}^{\top} \int_{t-T_{i j}(t)}^{t} \dot{\mathbf{z}}_{j}(\sigma) d \sigma\right. \\
& \left.-\frac{1}{2 \beta_{i}} \sum_{j \in \mathcal{N}_{i}} a_{i j}\left(T_{i j}^{* 2} v_{j}^{2}-T_{i j}^{*} \int_{t-T_{i j}^{*}}^{t} v_{j}^{2}(\sigma) d \sigma\right)\right] .
\end{aligned}
$$

where, to obtain the last equation, we used the fact that

$\frac{1}{2} \sum_{i \in \bar{N}} \sum_{j \in \mathcal{N}_{i}} a_{i j}\left(\dot{\overline{\mathbf{z}}}_{i}-\dot{\overline{\mathbf{z}}}_{j}\right)^{\top}\left(\overline{\mathbf{z}}_{i}-\overline{\mathbf{z}}_{j}\right)=\sum_{i \in \bar{N}} \sum_{j \in \mathcal{N}_{i}} a_{i j} \dot{\overline{\mathbf{z}}}_{i}^{\top}\left(\overline{\mathbf{z}}_{i}-\overline{\mathbf{z}}_{j}\right)$.

The latter holds under Assumption A1 $-c f$. [2, Lemma 6.1] and since

$$
\overline{\mathbf{z}}_{j}-\overline{\mathbf{z}}_{j}\left(t-T_{i j}(t)\right)=\int_{t-T_{i j}(t)}^{t} \dot{\overline{\mathbf{z}}}_{j}(\sigma) d \sigma .
$$

Next, we apply Young's and Cauchy-Schwarz' inequalities on the second right-hand-term of (17) to obtain

$$
\begin{aligned}
& -\dot{\mathbf{z}}_{i}^{\top} \int_{t-T_{i j}(t)}^{t} \dot{\mathbf{z}}_{j}(\sigma) d \sigma \leq \frac{\beta_{i}}{2}\left|\dot{\mathbf{z}}_{i}\right|^{2}+\frac{1}{2 \beta_{i}}\left|\int_{t-T_{i j}(t)}^{t} \dot{\mathbf{z}}_{j}(\sigma) d \sigma\right|^{2} \\
& \leq \frac{\beta_{i}}{2}\left|\dot{\mathbf{z}}_{i}\right|^{2}+\frac{T_{i j}^{*}}{2 \beta_{i}} \int_{t-T_{i j}^{*}}^{t}\left|\dot{\mathbf{z}}_{j}(\sigma)\right|^{2} d \sigma
\end{aligned}
$$

for any $\beta_{i}>0$. Now, since $\boldsymbol{\varphi}_{i}^{\top} \boldsymbol{\varphi}_{i}=1$ then $\left|\dot{\mathbf{z}}_{i}\right|^{2}=v_{i}^{2}$. Thus,

$$
\dot{\mathcal{V}} \leq-\sum_{i \in \bar{N}}\left[\left(\frac{d_{v i}}{p_{v i}}-\frac{\beta_{i}}{2} l_{i i}\right) v_{i}^{2}-\sum_{j \in \mathcal{N}_{i}} a_{i j} \frac{T_{i j}^{* 2}}{2 \beta_{i}} v_{j}^{2}\right],
$$

where $l_{i i}:=\sum_{j \in \mathcal{N}_{i}} a_{i j}$ is the $i$ th-diagonal element of the Laplacian matrix.

Following [19] and defining $\mathbf{s}\left(v_{i}^{2}\right):=\operatorname{col}\left[v_{1}^{2}, \ldots, v_{N}^{2}\right]$ and

$$
\Psi=\left[\begin{array}{cccc}
\frac{d_{v 1}}{p_{v 1}}-\frac{\beta_{1}}{2} l_{11} & -\frac{T_{21}^{* 2}}{2 \beta_{1}} a_{12} & \ldots & -\frac{T_{N 1}^{* 2}}{2 \beta_{1}} a_{1 N} \\
-\frac{T_{12}^{* 2}}{2 \beta_{2}} a_{21} & \frac{d_{v 2}}{p_{v 2}}-\frac{\beta_{2}}{2} l_{22} & \ldots & -\frac{T_{N 2}^{* 2}}{2 \beta_{2}} a_{2 N} \\
\vdots & \vdots & \ddots & \vdots \\
-\frac{T_{1 N}^{* 2}}{2 \beta_{N}} a_{N 1} & -\frac{T_{2 N}^{* 2}}{2 \beta_{N}} a_{N 2} & \ldots & \frac{d_{v N}}{p_{v N}}-\frac{\beta_{N}}{2} l_{N N}
\end{array}\right],
$$

we can write $\dot{\mathcal{V}} \leq-\mathbf{1}_{N}^{\top} \boldsymbol{\Psi} \mathbf{s}\left(v_{i}^{2}\right)$ or, equivalently,

$$
\dot{\mathcal{V}} \leq-\sum_{i \in \bar{N}}\left(\frac{d_{v i}}{p_{v i}}-\sum_{j \in \mathcal{N}_{i}} a_{i j}\left(\frac{\beta_{i}}{2}+\frac{T_{i j}^{* 2}}{2 \beta_{j}}\right)\right) v_{i}^{2} .
$$

Setting $d_{v i}$ such that (12) holds, it follows that there exists $\lambda_{i}>0$ such that $\dot{\mathcal{V}} \leq-\sum_{i \in \bar{N}} \lambda_{i} v_{i}^{2}$. Thus $v_{i} \in \mathcal{L}_{2}$. Further, since $\mathcal{V}$ is positive definite and radially unbounded with regards to $v_{i}$ and $\overline{\mathbf{z}}_{i}-\overline{\mathbf{z}}_{j}$, it follows that $v_{i}(t)$ and $\overline{\mathbf{z}}_{i}(t)-\overline{\mathbf{z}}_{j}(t)$ are bounded. Furthermore, since $\varphi_{i}$ is bounded for all $\theta_{i}$, from (14), it follows that $\dot{v}_{i}$ is also bounded. In addition, boundedness of $\mathbf{e}_{z i}$ is established with the fact that $v_{i} \in \mathcal{L}_{2}$ and $\overline{\mathbf{z}}_{i}-\overline{\mathbf{z}}_{j} \in \mathcal{L}_{\infty}$.

Stage 1b) Boundedness of $\omega_{i}(t)$ and $e_{\theta_{i}}(t)$ : In order to establish boundedness of $\omega_{i}$ we first consider $\Sigma_{\omega}$ subject to $\gamma_{i}=0$ and the Lyapunov-Krasovskii functional

$$
\begin{aligned}
& \mathcal{W}:=\frac{1}{2} \sum_{i \in \bar{N}} b_{i}\left(\theta_{i}-\theta_{\ell}\right)^{2}+ \\
& \sum_{i \in \bar{N}}\left[\frac{I_{i}}{2 p_{\omega i}} \omega_{i}^{2}+\frac{1}{4} \sum_{j \in \mathcal{N}_{i}} a_{i j}\left(\theta_{i}-\theta_{j}\right)^{2}\right. \\
&\left.+\frac{1}{2 \alpha_{i}} \sum_{j \in \mathcal{N}_{i}} a_{i j} T_{i j}^{*} \int_{-T_{i j}^{*}}^{0} \int_{t+\sigma}^{t} \omega_{j}^{2}(\eta) d \eta d \sigma\right],
\end{aligned}
$$

where $\alpha_{i}>0$. Proceeding like for the computation of the bound on $\dot{\mathcal{V}}$, we obtain

$$
\dot{\mathcal{W}} \leq-\sum_{i \in \bar{N}}\left[\left(\frac{d_{\omega i}}{p_{\omega i}}-\frac{\alpha_{i}}{2} l_{i i}\right) \omega_{i}^{2}-\sum_{j \in \mathcal{N}_{i}} a_{i j} \frac{T_{i j}^{* 2}}{2 \alpha_{i}} \omega_{j}^{2}\right],
$$

hence,

$$
\dot{\mathcal{W}} \leq-\sum_{i \in \bar{N}}\left(\frac{d_{\omega i}}{p_{\omega i}}-\sum_{j \in \mathcal{N}_{i}} a_{i j}\left(\frac{\alpha_{i}}{2}+\frac{T_{i j}^{* 2}}{2 \delta_{j}}\right)\right) \omega_{i}^{2} .
$$

Then, setting $d_{\omega i}$ such that (13) holds, it follows that there exists $\lambda_{i}>0$ such that $\dot{\mathcal{W}} \leq-\sum_{i \in \bar{N}} \lambda_{i} \omega_{i}^{2}$. Thus $\omega_{i} \in \mathcal{L}_{2}$. Further, since $\mathcal{W}$ is positive definite and radially unbounded with regards to $\omega_{i}$ and $\theta_{i}-\theta_{j}$, then these signals are bounded. Which, in turn, imply that $\dot{\omega}_{i} \in \mathcal{L}_{\infty}$. Invoking Barbălat's Lemma it is established that $\lim _{t \rightarrow \infty} \omega_{i}(t)=0$. This implies that

$$
\lim _{t \rightarrow \infty} \int_{0}^{t} \dot{\omega}_{i}(s) d s=\omega_{i}(0) \text {. }
$$


On the other hand, differentiating on both sides of the second equation in (15) (with $\gamma_{i}=0$ ) we see that $\ddot{\omega}_{i}$ is bounded so $\dot{\omega}_{i}$ is uniformly continuous. It follows, after Barbălat's Lemma, that $\dot{\omega}_{i} \rightarrow 0$.

Remark 2 Similar steps lead to concluding that $\dot{v}_{i} \rightarrow 0$ too. Now, once more from (15), it follows that

$$
\lim _{t \rightarrow \infty} b_{i}\left(\theta_{i}(t)-\theta_{\ell}\right)+\sum_{j \in \mathcal{N}_{i}} a_{i j}\left(\theta_{i}(t)-\theta_{j}\left(t-T_{i j}(t)\right)\right)=0,
$$

Considering all the $N$ elements we have that

$$
\lim _{t \rightarrow \infty} \mathbf{B}\left(\theta(t)-\mathbf{1}_{N} \otimes \theta_{\ell}\right)+\mathbf{L} \theta(t)=\mathbf{0},
$$

and because $\mathbf{L} \mathbf{1}_{N}=\mathbf{0}$ then $\lim _{t \rightarrow \infty} \mathbf{L}_{\ell}\left(\theta(t)-\mathbf{1}_{N} \otimes \theta_{\ell}\right)=\mathbf{0}$, where $\mathbf{L}_{\ell}$ is defined in Lemma 1 . Further, since $\mathbf{A} \mathbf{2}$ ensures, by Lemma 1, that $\mathbf{L}_{\ell}$ is of full-rank then $\lim \theta(t)=\mathbf{1}_{N} \otimes \theta_{\ell}$.

If $\gamma \not \equiv 0$, because $\gamma_{i} \in \mathcal{L}_{\infty}$ and $\Sigma_{\omega}$ is an asymptotically stable linear time-varying system with uniformly bounded time-delays then $\dot{\omega}_{i}, \omega_{i} \in \mathcal{L}_{\infty}$, by Proposition 3 in [20]. Thus $e_{\theta i}$ and $\theta_{i}-\theta_{\ell}$ are also bounded. This finishes Stage 1) of the proof.

Stage 2) Convergence. We have $v_{i} \in \mathcal{L}_{2} \cap \mathcal{L}_{\infty}$ and $\dot{v}_{i} \in$ $\mathcal{L}_{\infty}$. This implies, by Barbălat's Lemma, that $v_{i} \rightarrow 0$ and, in turn, that $\dot{v}_{i} \rightarrow 0$. Therefore, from the second equation in (14), we conclude that

$$
\lim _{t \rightarrow \infty} \boldsymbol{\varphi}_{i}(t)^{\top}\left[\overline{\mathbf{z}}_{i}(t)-\overline{\mathbf{z}}_{\ell}(t)+\mathbf{e}_{z i}(t)\right]=0 .
$$

However, note that $\varphi_{i}^{\top}\left[\overline{\mathbf{z}}_{i}-\overline{\mathbf{z}}_{\ell}+\mathbf{e}_{z i}\right]=0$ has many solutions other than $\left[\overline{\mathbf{z}}_{i}-\overline{\mathbf{z}}_{\ell}+\mathbf{e}_{z i}\right]=0$. Therefore, it must also be established that

$$
\lim _{t \rightarrow \infty} \boldsymbol{\varphi}_{i}(t)^{\top \perp}\left[\overline{\mathbf{z}}_{i}(t)-\overline{\mathbf{z}}_{\ell}(t)+\mathbf{e}_{z i}(t)\right]=0
$$

in order to conclude that

$$
\lim _{t \rightarrow \infty}\left(\mathbf{L}_{\ell} \otimes \mathbf{I}_{2}\right)\left(\overline{\mathbf{z}}(t)-\mathbf{1}_{N} \otimes \overline{\mathbf{z}}_{\ell}\right)=0 .
$$

This follows from applying successively Barbălat's Lemma to conclude that $\dot{f}(t) \boldsymbol{\varphi}_{i}(t)^{\top \perp}\left[\overline{\mathbf{z}}_{i}(t)-\overline{\mathbf{z}}_{\ell}(t)+\mathbf{e}_{z i}(t)\right] \rightarrow 0$ and using the assumption that $\dot{f}(t) \nrightarrow 0$. The detail of these lengthy computations is omitted due to space constraints.

\section{Simulation Results}

To illustrate the performance of the controllers proposed above, numerical simulations were performed in Simulink ${ }^{\circledR}$ of Matlab ${ }^{\circledR}$. The case-study consists in a network of six differential-drive robots affected by time-varying communication delays (all considered equal, for simplicity). These are modelled by a Gaussian distribution function with a mean of $0.3 \mathrm{~s}$ and a variance of $0.03 \mathrm{~s}$. The robots are required to acquire an hexagonal formation with center at the coordinates $\left(x_{\ell}, y_{\ell}\right)=(1,1)$ and common leader orientation of $\pi / 3 \mathrm{rad}$. The initial velocities are all set to zero while the initial positions are provided in Table I. In the latter, the offsets $\left(\delta_{x i}, \delta_{y i}\right)$ are also showed.

The paths described by the robots on the plane are illustrated in Figure 2. It may be appreciated there in that

\begin{tabular}{c|cccccc|} 
ROBOT & 1 & 2 & 3 & 4 & 5 & 6 \\
\hline$x_{i}(0)$ & 5 & 7 & 7 & 3 & 1 & 1 \\
$y_{i}(0)$ & 2 & 5.5 & 3.5 & 2 & 3.5 & 5.5 \\
$\theta_{i}(0)$ & 0 & $-\pi / 4$ & $-\pi / 2$ & $\pi / 4$ & $\pi / 2$ & $\pi / 4$ \\
\hline$\delta_{x i}$ & 2 & 1 & -1 & -2 & -1 & 1 \\
$\delta_{y i}$ & 0 & 2 & 2 & 0 & -2 & -2 \\
\hline
\end{tabular}

TABLE I

INITIAL POSITIONS AND RELATIVE DESIRED TRANSLATION OF THE $i$ TH-ROBOT WITH REGARDS TO THE BARYCENTER.

the formation goal is achieved. The final orientations of the robots are depicted by pointing arrows, making evident as well, the achievement of the consensus-in-orientation goal. Note that all orientations match the leader's $\theta_{\ell}=\pi / 3$.

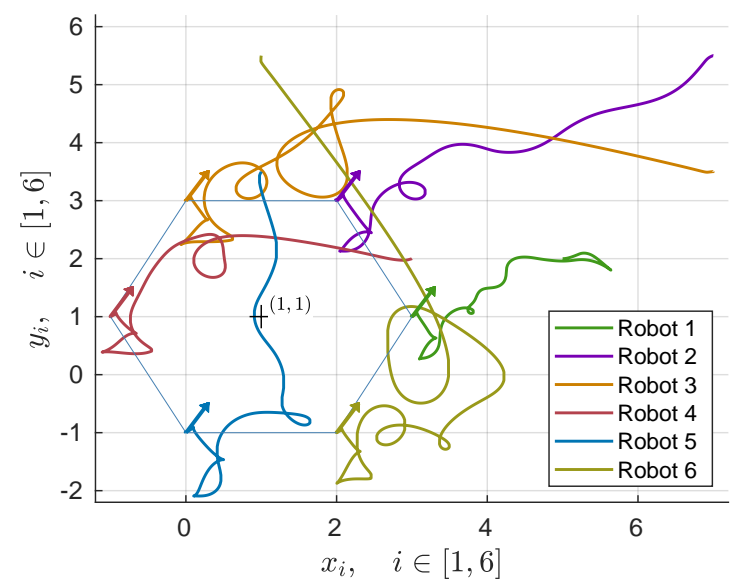

Fig. 2. Robots' paths on the plane, achieving consensus to the leader's position $(1,1)$

The control gains were set to $d_{v i}=800, p_{v i}=300, b_{i}=$ $1, d_{\omega i}=600, p_{\omega i}=300$, and $k_{\gamma i}=200$ while the function $f(t)$ was defined to be periodic, as $f(t):=7 \sin (0.2 t)$. It is to be noted that $f$ does not need to be periodic, however, this is ab ad hoc manner to satisfy the condition that $\dot{f} \nrightarrow \rightarrow 0$. In general, $f$ is chosen so that $\dot{f}$ be persistently exciting.

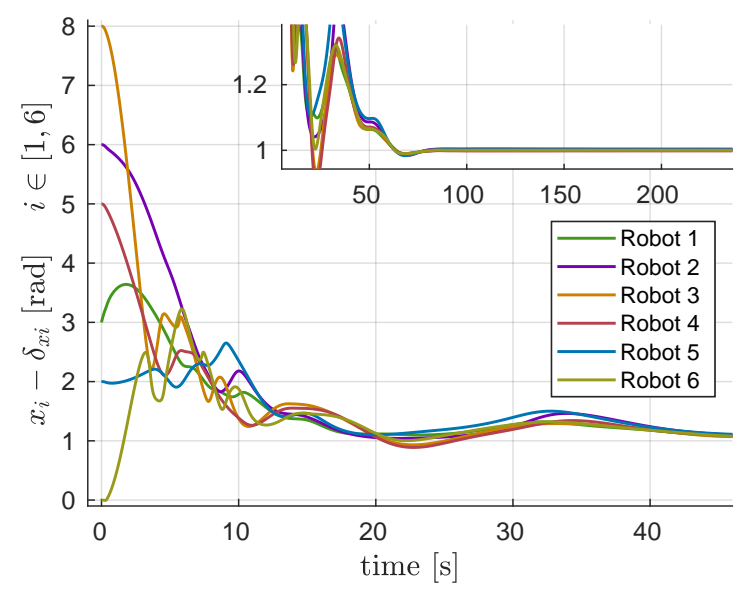

Fig. 3. Relative abscissae coordinates $\bar{x}_{i}$ against time

In Figures 3-5 are depicted the system's responses regard- 
ing the Cartesian coordinate errors $\bar{x}_{i}$ and $\bar{y}_{i}$ as well as the robots' individual orientations. It may be appreciated from these curves that not only all coordinates converge to the leader's position and orientation, but during the transients, they all converge to each other, hence achieving consensus.

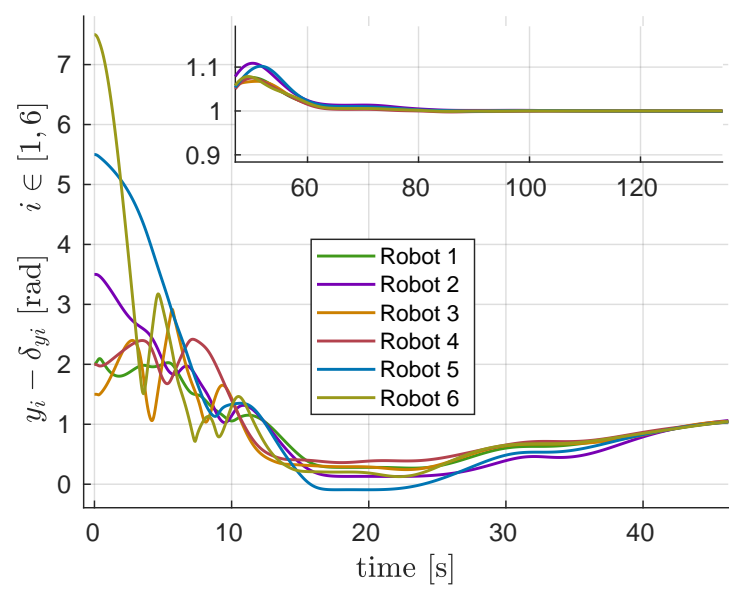

Fig. 4. Relative ordinate coordinates $\bar{y}_{i}$ against time

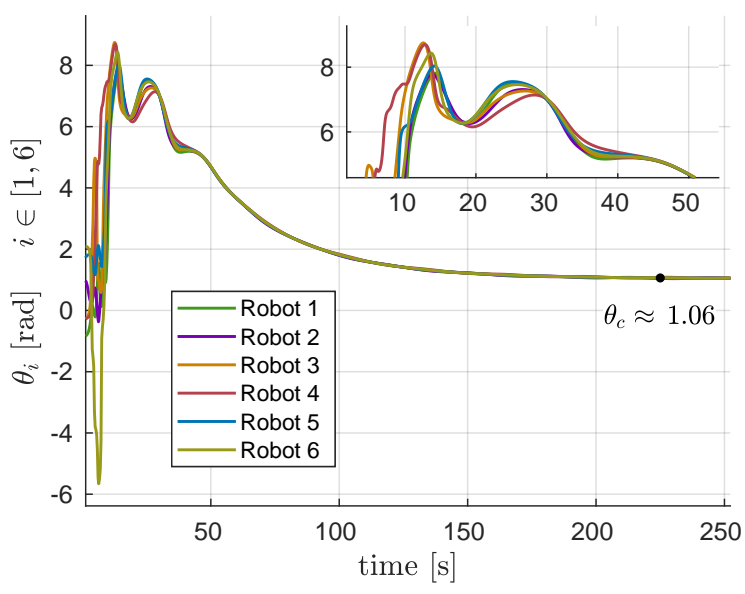

Fig. 5. Orientations of the robots achieving consensus and converging to $\pi / 3 \mathrm{rad}$.

\section{CONCLUding REMARKS}

We presented a controller for consensus formation and setpoint stabilization of a group of robots communicating under the effect of time-varying delays. Contrary to trajectorytracking formation controllers for nonholonomic vehicles, where the desired trajectory is restricted to be a solution of the nonholonomic restriction, the difficulty behind the solution of formation-consensus problems resides in the fact that the leader's position is arbitrary. This problem is sorted out using a time-varying controller with persistency of excitation. Although this condition is necessary in the present setting, it has the disadvantage that the response may be affected by an undesirable oscillatory behaviour. Therefore, a careful controller tunning is needed. On the other hand, the controller that we presented is entirely decentralized and ensures consensus and stabilization both in the Cartesian positions and orientations. Further research is aimed at relaxing the assumption that the whole state, notably the velocities, are available from measurement.

\section{REFERENCES}

[1] W. Ren and R. W. Beard, Distributed consensus in multivehicle cooperative control. Springer verlag, 2005.

[2] Y. Cao and W. Ren, Distributed Coordination of Multi-agent Networks: Emergent Problems, Models, and Issues. Springer-Verlag, 2011.

[3] H. Wang, "Consensus of networked mechanical systems with communication delays: A unified framework," IEEE Transactions on Automatic Control, vol. 59, no. 6, pp. 1571-1576, 2014.

[4] T. Hatanaka, N. Chopra, M. Fujita, and M. Spong, Passivity-Based Control and Estimation in Networked Robotics. Communications and Control Engineering, Springer, 2015.

[5] D. A. Lizárraga., "Obstructions to the existence of universal stabilizers for smooth control systems," Mathematics of Control, Signals and Systems, vol. 16, p. 255-277, 2004.

[6] T. Uzunovic and A. Sabanovic, "Formation control of differentialdrive mobile robots in the framework of functionally related systems," in IECON 2015 - 41st Annual Conference of the IEEE Industrial Electronics Society, pp. 002620-002625, 2015.

[7] Y. Q. Chen and Z. Wang, "Formation control: a review and a new consideration," in 2005 IEEE/RSJ International Conference on Intelligent Robots and Systems, pp. 3181-3186, Aug 2005.

[8] D. Dimarogonas and K. Kyriakopoulos, "On the rendezvous problem for multiple nonholonomic agents," Automatic Control, IEEE Transactions on, vol. 52, no. 5, pp. 916-922, 2007.

[9] Z. Lin, B. Francis, and M. Maggiore, "Necessary and sufficient graphical conditions for formation control of unicycles," Automatic Control, IEEE Transactions on, vol. 50, no. 1, pp. 121-127, 2005.

[10] C. Yang, W. Xie, C. Lei, and B. Ma, "Smooth time-varying formation control of multiple nonholonomic agents," in Proceedings of the 2015 Chinese Intelligent Systems Conference, pp. 283-291, Springer, 2016.

[11] A. Ajorlou, M. Asadi, M. Aghdam, and S. Blouin, "Distributed consensus control of unicycle agents in the presence of external disturbances," Systems \& Control Letters, vol. 82, pp. 86-90, 2015.

[12] Z. Peng, G. Wen, A. Rahmani, and Y. Yu, "Distributed consensusbased formation control for multiple nonholonomic mobile robots with a specified reference trajectory," International Journal of Systems Science, vol. 46, no. 8, pp. 1447-1457, 2015.

[13] A. Bautista-Castillo, C. Lopez-Franco, and E. Nuño, "Consensusbased formation control for multiple nonholonomic robots," in 2016 IEEE International Autumn Meeting on Power, Electronics and Computing (ROPEC), IEEE, 2016.

[14] M. Maghenem, A. Loría, and E. Panteley, "A robust $\delta$-persistently exciting controller for leader-follower tracking-agreement of multiple vehicles," European J. of Control, vol. 40, pp. 1-12, 2018.

[15] M. Maghenem, A. Bautista-Castillo, E. Nuño, A. Loría, and E. Panteley, "Consensus-based formation control of nonholonomic robots using a strict Lyapunov function," IFAC-PapersOnLine, vol. 50, no. 1, pp. 2439-2444, 2017. Presented at IFAC World Congress 2017, Toulouse, France.

[16] W. Dong and J. Farrell, "Consensus of multiple nonholonomic systems," in Decision and Control, 2008. CDC 2008. 47th IEEE Conference on, pp. 2270-2275, IEEE, 2008.

[17] Y. Hong, J. Hu, and L. Gao, "Tracking control for multi-agent consensus with an active leader and variable topology," Automatica, vol. 42, no. 7, pp. 1177-1182, 2006.

[18] A. Loría, E. Panteley, and A. Teel, "A new persistency-of-excitation condition for UGAS of NLTV systems: Application to stabilization of nonholonomic systems," in Proc. 5th. European Contr. Conf., (Karlsrühe, Germany), pp. 1363-1368, 1999.

[19] E. Nuño, I. Sarras, and L. Basañez, "Consensus in networks of nonidentical Euler-Lagrange systems using P+d controllers," IEEE Transactions on Robotics, vol. 26, no. 6, pp. 1503-1508, 2013.

[20] H. Wang, "Integral-cascade framework for consensus of networked Lagrangian systems," tech. rep., 2018. 